\title{
An Analysis of Brand Selection
}

\author{
Kazuhiro Takeyasu† \\ College of Business Administration, Tokoha University, \\ 325 Oobuchi, Fuji City, Shizuoka, 417-0801, \\ Japan
}

\begin{abstract}
It is often observed that consumers select upper class brand when they buy next time. Suppose that former buying data and current buying data are gathered. Also suppose that upper brand is located upper in the variable array. Then the transition matrix becomes upper triangular matrix under the supposition that former buying variables are set input and current buying variables are set output. Takeyasu et al. analyzed the brand selection and its matrix structure before. In that paper, products of one genre are analyzed. In this paper, brand selection among multiple genre and its matrix structure are analyzed. Taking an automobile for example, customer brand selection from company $A$ to $B$ or company $A$ to $C$ can be made clear utilizing above stated method. We can confirm not only the preference shift among brands but also the preference shift among companies. This enables building marketing strategy for automobile company much easier. Analyzing such structure provides useful applications. Thus, this proposed approach enables to make effective marketing plan and/or establishing new brand.
\end{abstract}

Keywords — brand selection; matrix structure; brand position

\section{INTRODUCTION}

Marketing analysis is one of the "never ending themes" because there arise lots of events and new trend in the market and society. There are many themes to be investigated and the analyses may be utilized for marketing plan etc. In this paper, we focus on the brand selection by consumers.

It is often observed that consumers select upper class brand when they buy next time after they are bored to use current brand. Suppose that former buying data and current buying data are gathered. Also suppose that upper brand is located upper in the variable array. Then the transition matrix becomes upper triangular matrix under the supposition that former buying variables are set input and current buying variables are set output. The analysis of the brand selection in the same brand group is analyzed by Takeyasu et al. [6].

In this paper, we expand this scheme to products of multiple genres. For example, we consider the case of necklace. If she is accustomed to use necklace, she would buy higher priced necklace. On the other hand, she may buy bracelet or earring for her total coordination in fashion. Hearing from the retailer, both can be seen in selecting upper class brand and selecting another genre product.

Therefore, this analysis is very meaningful for the practical use, which occurs actually. If transition matrix is identified, we can make various analyses using it and s-step forecasting can be executed. Unless planners for products notice its brand position whether it is upper or lower than other products,

\author{
Yuki Higuchi \\ Faculty of Business Administration, Setsunan University, \\ 17-8 Ikeda-nakamachi, Neyagawa, Osaka, 572-8508, \\ Japan
}

matrix structure makes it possible to identify those by calculating consumers' activities for brand selection. Thus, this proposed approach makes it effective to execute marketing plan and/or establish new brand.

Quantitative analysis concerning brand selection has been executed by Yamanaka [5], Takahashi et al.[4]. Yamanaka[5] examined purchasing process by Markov Transition Probability with the input of advertising expense. Takahashi et al.[4] made analysis by the Brand Selection Probability model using logistics distribution. Takeyasu et al.[6] analyzed the preference shift of customer brand selection in the case of automobile.

Takeyasu et al.[7] analyzed the preference shift of customer brand selection for a single brand group. In this paper, we try to expand this scheme to products of multiple genres, and various analyses is executed. Actually, this scheme can often be seen. Such research is quite a new one.

Hereinafter, matrix structure for a single brand group is clarified for the selection of brand in section 2. Expansion to multiple brand selection is executed and analyzed in section 3 . s-step forecasting is stated in section 4. Numerical calculation is executed in section 5. Application of this method is extended in section 6 .

\section{Brand Selection And Its Matrix Structure}

\section{A. Upper shift of Brand selection}

Now, suppose that $x$ is the most upper class brand, $y$ is the second upper class brand, and $z$ is the lowest class brand. Consumer's behavior of selecting brand might be $z \rightarrow y$, $y \rightarrow x, z \rightarrow x$ etc. $x \rightarrow z$ might be few.

Suppose that $x$ is current buying variable, and $x_{b}$ is previous buying variable. Shift to $x$ is executed from

$$
x_{b}, y_{b} \text {, or } z_{b} \text {. }
$$

Therefore, $x$ is stated in the following equation. $a_{i j}$ Represents the transition probability from $j$-th to $i$-th brand.

$$
\begin{gathered}
x=a_{11} x_{b}+a_{12} y_{b}+a_{13} z_{b} \\
\text { Similarly, } \\
y=a_{22} y_{b}+a_{23} z_{b} \\
\text { and }
\end{gathered}
$$




$$
z=a_{33} z_{b}
$$

These are re-written as follows.

$$
\left(\begin{array}{l}
x \\
y \\
z
\end{array}\right)=\left(\begin{array}{ccc}
a_{11} & a_{12} & a_{13} \\
0 & a_{22} & a_{23} \\
0 & 0 & a_{33}
\end{array}\right)\left(\begin{array}{c}
x_{b} \\
y_{b} \\
z_{b}
\end{array}\right)
$$

\section{Set}

$$
\mathbf{X}=\left(\begin{array}{c}
x \\
y \\
z
\end{array}\right), \mathbf{A}=\left(\begin{array}{ccc}
a_{11} & a_{12} & a_{13} \\
0 & a_{22} & a_{23} \\
0 & 0 & a_{33}
\end{array}\right), \mathbf{X}_{\mathbf{b}}=\left(\begin{array}{c}
x_{b} \\
y_{b} \\
z_{b}
\end{array}\right)
$$

Then, $\mathbf{X}$ is represented as follows.

$$
\mathbf{X}=\mathbf{A} \mathbf{X}_{\mathbf{b}}
$$

Here,

$$
\mathbf{X} \in \mathbf{R}^{3}, \mathbf{A} \in \mathbf{R}^{3 \times 3}, \mathbf{X}_{\mathbf{b}} \in \mathbf{R}^{3}
$$

$\mathbf{A}$ is an upper triangular matrix.

To examine this, generating the following data, which all consist of the data in which the transition is made from lower brand to upper brand,

$$
\begin{aligned}
& \mathbf{X}^{\mathbf{i}}=\left(\begin{array}{l}
1 \\
0 \\
0
\end{array}\right) \quad\left(\begin{array}{l}
1 \\
0 \\
0
\end{array}\right) \quad \ldots \quad\left(\begin{array}{l}
0 \\
1 \\
0
\end{array}\right) \\
& \mathbf{X}_{\mathbf{b}}^{\mathbf{i}}=\left(\begin{array}{l}
0 \\
1 \\
0
\end{array}\right) \quad\left(\begin{array}{l}
1 \\
0 \\
0
\end{array}\right) \quad \ldots \quad\left(\begin{array}{l}
0 \\
0 \\
1
\end{array}\right) \\
& i=1, \quad 2 \quad \ldots \quad N
\end{aligned}
$$

Parameter can be estimated by using least square method.

Suppose

$$
\begin{gathered}
\mathbf{X}^{i}=\mathbf{A} \mathbf{X}_{\mathbf{b}}^{i}+\boldsymbol{\varepsilon}^{i} \\
\text { Where } \\
\varepsilon^{i}=\left(\begin{array}{c}
\varepsilon_{1}^{i} \\
\varepsilon_{2}^{i} \\
\varepsilon_{3}^{i}
\end{array}\right) \quad i=1,2, \cdots, N
\end{gathered}
$$

and minimize following $J$

$$
J=\sum_{i=1}^{N} \boldsymbol{\varepsilon}^{i T} \boldsymbol{\varepsilon}^{i} \rightarrow \text { Min }
$$

$\hat{\mathbf{A}}$ Which an estimated value is of $\mathbf{A}$ is obtained as follows.

$$
\hat{\mathbf{A}}=\left(\sum_{i=1}^{N} \mathbf{X}^{i} \mathbf{X}_{\mathbf{b}}^{i T}\right)\left(\sum_{i=1}^{N} \mathbf{X}_{\mathbf{b}}^{i} \mathbf{X}_{\mathbf{b}}^{i T}\right)^{-1}
$$

In the data group which all consist of the data in which the transition is made from lower brand to upper brand, estimated value $\hat{\mathbf{A}}$ should be upper triangular matrix. If the following data which shift to lower brand are added only a few in equation (3) and (4),

$$
\mathbf{X}^{i}=\left(\begin{array}{l}
0 \\
1 \\
0
\end{array}\right), \quad \mathbf{X}_{\mathbf{b}}^{i}=\left(\begin{array}{l}
1 \\
0 \\
0
\end{array}\right)
$$

$\hat{\mathbf{A}}$ would contain minute items in the lower part of triangle.

\section{B. Sorting brand ranking by re-arranging row}

In a general data, variables may not be in order as $x, y, z$ . In that case, large and small value lie scattered in

$\hat{\mathbf{A}}$. But re-arranging this, we can set in order by shifting row. The large value parts are gathered in the upper triangular matrix, and the small value parts are gathered in the lower triangular matrix.

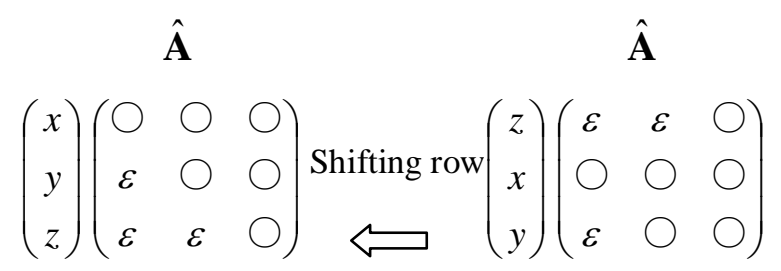

\section{Matrix structure under the case skipping intermediate class brand is skipped}

It is often observed that some consumers select the most upper class brand from the most lower class brand and skip selecting the intermediate class brand.

We suppose $v, w, x, y, z$ brands (suppose they are laid from upper position to lower position as $v>w>x>y>z$ ). In the above case, selection shifts would be:

$$
\begin{aligned}
& v \leftarrow z \\
& v \leftarrow y
\end{aligned}
$$

Suppose they do not shift to $y, x, w$ from $z$, to $x, w$ from $y$, and to $w$ from $x$, then Matrix structure would be as follows. 


$$
\left(\begin{array}{l}
v \\
w \\
x \\
y \\
z
\end{array}\right)=\left(\begin{array}{ccccc}
a_{11} & a_{12} & a_{13} & a_{14} & a_{15} \\
0 & a_{22} & 0 & 0 & 0 \\
0 & 0 & a_{33} & 0 & 0 \\
0 & 0 & 0 & a_{44} & 0 \\
0 & 0 & 0 & 0 & a_{55}
\end{array}\right)\left(\begin{array}{c}
v_{b} \\
w_{b} \\
x_{b} \\
y_{b} \\
z_{b}
\end{array}\right)
$$

We confirm this by the numerical example in section 4 .

\section{Expansion Of The Model To Multiple Genre PRODUCTS}

Expanding Eq.(2) to multiple genre products, we obtain the following equations. First of all, we state the generalized model of Eq.(2).

$$
\begin{gathered}
\mathbf{X}=\mathbf{A X}_{\mathbf{b}} \\
\mathbf{X}=\left(\begin{array}{c}
x^{1} \\
x^{2} \\
\vdots \\
x^{p}
\end{array}\right), \mathbf{X}_{\mathbf{b}}=\left(\begin{array}{c}
x_{b}^{1} \\
x_{b}^{2} \\
\vdots \\
x_{b}^{p}
\end{array}\right) \\
\mathbf{A}=\left(\begin{array}{cccc}
a_{11} & a_{12} & \cdots & a_{1 p} \\
a_{21} & a_{22} & \cdots & a_{2 p} \\
\vdots & \vdots & & \vdots \\
a_{p 1} & a_{p 2} & \cdots & a_{p p}
\end{array}\right) \\
\text { Here }
\end{gathered}
$$

$$
\mathbf{X} \in \mathbf{R}^{p}, \mathbf{A} \in \mathbf{R}^{p \times p}, \mathbf{X}_{\mathbf{b}} \in \mathbf{R}^{p}
$$

If the brand selection is executed towards upper class, then A becomes as follows.

$$
\mathbf{A}=\left(\begin{array}{cccc}
a_{11} & a_{12} & \cdots & a_{1 p} \\
0 & a_{22} & \cdots & a_{2 p} \\
\vdots & \vdots & & \vdots \\
0 & 0 & \cdots & a_{p p}
\end{array}\right)
$$

Expanding above equations to products of 3 genres, we obtain the following equations.

$$
\left(\begin{array}{l}
\mathbf{X} \\
\mathbf{Y} \\
\mathbf{Z}
\end{array}\right)=\left(\begin{array}{lll}
\mathbf{A}^{11} & \mathbf{A}^{12} & \mathbf{A}^{13} \\
\mathbf{A}^{21} & \mathbf{A}^{22} & \mathbf{A}^{23} \\
\mathbf{A}^{31} & \mathbf{A}^{32} & \mathbf{A}^{33}
\end{array}\right)\left(\begin{array}{c}
\mathbf{X}_{\mathbf{b}} \\
\mathbf{Y}_{\mathbf{b}} \\
\mathbf{Z}_{\mathbf{b}}
\end{array}\right)
$$

$$
\begin{gathered}
\mathbf{X}=\left(\begin{array}{c}
x^{1} \\
x^{2} \\
\vdots \\
x^{p}
\end{array}\right), \mathbf{X}_{\mathbf{b}}=\left(\begin{array}{c}
x_{b}^{1} \\
x_{b}^{2} \\
\vdots \\
x_{b}^{p}
\end{array}\right), \quad \mathbf{Y}=\left(\begin{array}{c}
y^{1} \\
y^{2} \\
\vdots \\
y^{q}
\end{array}\right), \\
\mathbf{Y}_{\mathbf{b}}=\left(\begin{array}{c}
y_{b}^{1} \\
y_{b}^{2} \\
\vdots \\
y_{b}^{q}
\end{array}\right), \mathbf{Z}=\left(\begin{array}{c}
z^{1} \\
z^{2} \\
\vdots \\
z_{b}^{r}
\end{array}\right), \mathbf{Z}_{\mathbf{b}}=\left(\begin{array}{c}
z_{b}^{2} \\
\vdots \\
z_{b}^{r}
\end{array}\right)
\end{gathered}
$$

$\mathbf{A}^{11}=\left(\begin{array}{cccc}a_{11}^{11} & a_{12}^{11} & \cdots & a_{1 p}^{11} \\ a_{21}^{11} & a_{22}^{11} & \cdots & a_{2 p}^{11} \\ \vdots & \vdots & & \vdots \\ a_{p 1}^{11} & a_{p 2}^{11} & \cdots & a_{p p}^{11}\end{array}\right)$,

$\mathbf{A}^{12}=\left(\begin{array}{cccc}a_{11}^{12} & a_{12}^{12} & \cdots & a_{1 q}^{12} \\ a_{21}^{12} & a_{22}^{12} & \cdots & a_{2 q}^{12} \\ \vdots & \vdots & & \vdots \\ a_{p 1}^{12} & a_{p 2}^{12} & \cdots & a_{p q}^{12}\end{array}\right)$,

$\mathbf{A}^{13}=\left(\begin{array}{cccc}a_{11}^{13} & a_{12}^{13} & \cdots & a_{1 r}^{13} \\ a_{21}^{13} & a_{22}^{13} & \cdots & a_{2 r}^{13} \\ \vdots & \vdots & & \vdots \\ a_{p 1}^{13} & a_{p 2}^{13} & \cdots & a_{p r}^{13}\end{array}\right)$,

$\mathbf{A}^{21}=\left(\begin{array}{cccc}a_{11}^{21} & a_{12}^{21} & \cdots & a_{1 p}^{21} \\ a_{21}^{21} & a_{22}^{21} & \cdots & a_{2 p}^{21} \\ \vdots & \vdots & & \vdots \\ a_{q 1}^{21} & a_{q 2}^{21} & \cdots & a_{q p}^{21}\end{array}\right)$,

$\mathbf{A}^{23}=\left(\begin{array}{cccc}a_{11}^{23} & a_{12}^{23} & \cdots & a_{1 r}^{23} \\ a_{21}^{23} & a_{22}^{23} & \cdots & a_{2 r}^{23} \\ \vdots & \vdots & & \vdots \\ a_{q 1}^{23} & a_{q 2}^{23} & \cdots & a_{q r}^{23}\end{array}\right)$,

Where 


$$
\begin{aligned}
\mathbf{A}^{31} & =\left(\begin{array}{cccc}
a_{11}^{31} & a_{12}^{31} & \cdots & a_{1 p}^{31} \\
a_{21}^{31} & a_{22}^{31} & \cdots & a_{2 p}^{31} \\
\vdots & \vdots & & \vdots \\
a_{r 1}^{31} & a_{r 2}^{31} & \cdots & a_{r p}^{31}
\end{array}\right), \\
\mathbf{A}^{32} & =\left(\begin{array}{cccc}
a_{11}^{32} & a_{12}^{32} & \cdots & a_{1 q}^{32} \\
a_{21}^{32} & a_{22}^{32} & \cdots & a_{2 q}^{32} \\
\vdots & \vdots & & \vdots \\
a_{r 1}^{32} & a_{r 2}^{32} & \cdots & a_{r q}^{32}
\end{array}\right), \\
\mathbf{A}^{33} & =\left(\begin{array}{cccc}
a_{11}^{33} & a_{12}^{33} & \cdots & a_{1 r}^{33} \\
a_{21}^{33} & a_{22}^{33} & \cdots & a_{2 r}^{33} \\
\vdots & \vdots & & \vdots \\
a_{r 1}^{33} & a_{r 2}^{33} & \cdots & a_{r r}^{33}
\end{array}\right)
\end{aligned}
$$

$\mathbf{X} \in \mathbf{R}^{p}, \mathbf{X}_{\mathbf{b}} \in \mathbf{R}^{p}, \mathbf{Y} \in \mathbf{R}^{q}, \mathbf{Y}_{\mathbf{b}} \in \mathbf{R}^{q}, \mathbf{Z} \in \mathbf{R}^{r}$,

$\mathbf{Z}_{\mathbf{b}} \in \mathbf{R}^{r}, \mathbf{A}^{11} \in \mathbf{R}^{p \times p}, \mathbf{A}^{12} \in \mathbf{R}^{p \times q}, \mathbf{A}^{13} \in \mathbf{R}^{p \times r}$,

$\mathbf{A}^{21} \in \mathbf{R}^{q \times p}, \mathbf{A}^{22} \in \mathbf{R}^{q \times q}, \mathbf{A}^{23} \in \mathbf{R}^{q \times r}$,

$\mathbf{A}^{31} \in \mathbf{R}^{r \times p}, \mathbf{A}^{32} \in \mathbf{R}^{r \times q}, \mathbf{A}^{33} \in \mathbf{R}^{r \times r}$

Re-writing Eq.(14) as :

$$
\mathbf{W}=\mathbf{A} \mathbf{W}_{\mathbf{b}}
$$

Then, the transition matrix $\mathbf{A}$ is derived as follows in the same way with Eq.(7).

$$
\begin{gathered}
\hat{\mathbf{A}}=\left(\sum_{i=1}^{N} \mathbf{W}^{i} \mathbf{W}_{\mathbf{b}}^{i T}\right)\left(\sum_{i=1}^{N} \mathbf{W}_{\mathbf{b}}^{i} \mathbf{W}_{\mathbf{b}}^{i T}\right)^{-1} \\
\mathbf{W}=\left(\begin{array}{c}
\mathbf{X} \\
\mathbf{Y} \\
\mathbf{Z}
\end{array}\right), \quad \mathbf{W}_{\mathbf{b}}=\left(\begin{array}{c}
\mathbf{X}_{\mathbf{b}} \\
\mathbf{Y}_{\mathbf{b}} \\
\mathbf{Z}_{\mathbf{b}}
\end{array}\right) \\
\mathbf{A}=\left(\begin{array}{lll}
\mathbf{A}^{11} & \mathbf{A}^{12} & \mathbf{A}^{13} \\
\mathbf{A}^{21} & \mathbf{A}^{22} & \mathbf{A}^{23} \\
\mathbf{A}^{31} & \mathbf{A}^{32} & \mathbf{A}^{33}
\end{array}\right) \\
\mathbf{W}^{i}=\mathbf{A} \mathbf{W}_{\mathbf{b}}^{i}+\boldsymbol{\varepsilon}^{i} \quad i=1,2, \cdots, N
\end{gathered}
$$

$$
\boldsymbol{\varepsilon}^{i}=\left(\begin{array}{c}
\varepsilon_{1}^{i} \\
\vdots \\
\varepsilon_{p}^{i} \\
\varepsilon_{p+1}^{i} \\
\vdots \\
\varepsilon_{p+q}^{i} \\
\varepsilon_{p+q+1}^{i} \\
\vdots \\
\varepsilon_{p+q+r}^{i}
\end{array}\right) \quad i=1,2, \cdots, N
$$

If the brand selection is executed towards upper class brand in the same genre, the transition matrix, for example

$\mathbf{A}_{11}, \mathbf{A}_{22}, \mathbf{A}_{33}$, become an upper triangular matrix as can be seen in 2. Suppose $\mathbf{X}$ as bracelet, $\mathbf{Y}$ as earring and $\mathbf{Z}$ as necklace. If we only see $\mathbf{Z}$, we can examine whether there is an upper brand shift in $\mathbf{A}_{33}$. But there is a case that brand selection is executed towards other genre products. There occurs brand selection shift from a certain brand level of $\mathbf{Z}$ to a certain brand level of $\mathbf{X}$ or $\mathbf{Y}$. For example, suppose there are five levels in each $\mathbf{X}, \mathbf{Y}, \mathbf{Z}$ and their levels include from bottom to top brand level. In that case, if there is a brand selection shift from the middle brand level in $\mathbf{Z}$ to another genre product, we can obtain interesting result by examining how the brand selection shift is executed toward the same level or upper level of another genre product. If we can see the trend of brand selection shift, we can foresee the brand selection shift towards another genre brand. Retailer can utilize the result of this to make effective marketing plan. We confirm this by the simple numerical example in 5 .

Next, we examine the case in brand groups. Matrices are composed by Block Matrix.

\section{IV. $S$-STEP FORECASTING}

Now, we see Eq.(14) in time series. Set $\mathbf{X}, \mathbf{Y}, \mathbf{Z}$ at time $n$ as :

$$
\mathbf{X}_{\mathbf{n}}=\left(\begin{array}{c}
x_{1}^{n} \\
x_{2}^{n} \\
\vdots \\
x_{p}^{n}
\end{array}\right), \mathbf{Y}_{\mathbf{n}}=\left(\begin{array}{c}
y_{1}^{n} \\
y_{2}^{n} \\
\vdots \\
y_{q}^{n}
\end{array}\right), \mathbf{Z}_{\mathbf{n}}=\left(\begin{array}{c}
z_{1}^{n} \\
z_{2}^{n} \\
\vdots \\
z_{r}^{n}
\end{array}\right)
$$

Then, Eq.(14) can be re-stated as :

$$
\left(\begin{array}{l}
\mathbf{X}_{\mathbf{n}} \\
\mathbf{Y}_{\mathbf{n}} \\
\mathbf{Z}_{\mathbf{n}}
\end{array}\right)=\left(\begin{array}{lll}
\mathbf{A}_{11} & \mathbf{A}_{12} & \mathbf{A}_{13} \\
\mathbf{A}_{21} & \mathbf{A}_{22} & \mathbf{A}_{23} \\
\mathbf{A}_{31} & \mathbf{A}_{32} & \mathbf{A}_{33}
\end{array}\right)\left(\begin{array}{c}
\mathbf{X}_{\mathbf{n}-\mathbf{1}} \\
\mathbf{Y}_{\mathbf{n}-\mathbf{1}} \\
\mathbf{Z}_{\mathbf{n}-\mathbf{1}}
\end{array}\right)
$$


Where suffix is written in the lower part of right hand side because there arises a multiplier in the equation of forecasting.

$S$-step forecasting is executed by the following equation.

$$
\left(\begin{array}{l}
\mathbf{X}_{\mathbf{n}+\mathbf{s}} \\
\mathbf{Y}_{\mathbf{n}+\mathbf{s}} \\
\mathbf{Z}_{\mathbf{n}+\mathbf{s}}
\end{array}\right)=\left(\begin{array}{lll}
\mathbf{A}_{11} & \mathbf{A}_{12} & \mathbf{A}_{13} \\
\mathbf{A}_{21} & \mathbf{A}_{22} & \mathbf{A}_{23} \\
\mathbf{A}_{31} & \mathbf{A}_{32} & \mathbf{A}_{33}
\end{array}\right)^{s}\left(\begin{array}{l}
\mathbf{X}_{\mathbf{n}} \\
\mathbf{Y}_{\mathbf{n}} \\
\mathbf{Z}_{\mathbf{n}}
\end{array}\right)
$$

\section{NUMERICAL EXAMPLE}

We consider the case of $p=q=r=4$ in 3. Suppose there are following customer preference shifts.

From the lower level of $\mathbf{Z}$ to middle or upper level of $\mathbf{Z}$

From the lower level of $\mathbf{Z}$ to lower, middle or upper level of $\mathbf{Y}$

From the middle level of $\mathbf{Z}$ to middle or upper level of $\mathbf{Y}$

From the upper level of $\mathbf{Z}$ to upper level of $\mathbf{Y}$

From the lower level of $\mathbf{Z}$ to lower, middle or upper level of $\mathbf{X}$ $\mathbf{X}$

From the middle level of $\mathbf{Z}$ to middle or upper level of

From the upper level of $\mathbf{Z}$ to upper level of $\mathbf{X}$

And also suppose that there are preference shifts which stay at the same level in $\mathbf{Z}$. For simplicity, preference shift of $\mathbf{X}, \mathbf{Y}$ stays at the same level within $\mathbf{X}$ and $\mathbf{Y}$. In these cases, we can assume that each block matrix in $\mathbf{A}$ of Eq.(21) becomes as follows.

$$
\mathbf{A}_{11}, \mathbf{A}_{22}: \text { Diagonal matrix }
$$

$$
\mathbf{A}_{12}, \mathbf{A}_{21}, \mathbf{A}_{31}, \mathbf{A}_{32}: \mathbf{0}
$$

$$
\mathbf{A}_{13}, \mathbf{A}_{23}, \mathbf{A}_{33}: \text { Upper triangular matrix }
$$

Now we suppose customer preference shifts as follows.

$\begin{array}{llll}\text { 1. } & \text { Jump from 4th to 3rd rank of } \mathbf{Z} & : & 2 \text { events } \\ \text { 2. } & \text { Jump from 4th to 2nd rank of } \mathbf{Z} & : & 1 \text { event } \\ \text { 3. } & \text { Jump from 2nd to 1st rank of } \mathbf{Z} & : & 2 \text { events } \\ \text { 4. } & \text { Stay at 4th rank of } \mathbf{Z} & : & 3 \text { events } \\ \text { 5. } & \text { Stay at 3rd rank of } \mathbf{Z} & : & 4 \text { events } \\ \text { 6. } & \text { Stay at 2nd rank of } \mathbf{Z} & : & 4 \text { events } \\ \text { 7. } & \text { Stay at 1st rank of } \mathbf{Z} & : & 2 \text { events } \\ \text { 8. } & \text { Jump from 4th rank of } \mathbf{Z} \text { to 3rd rank of } & : & 1 \text { event } \\ & \mathbf{Y} & \\ \text { 9. } & \text { Jump from 4th rank of } \mathbf{Z} \text { to 2nd rank of } & : & 1 \text { event } \\ & \mathbf{Y} & \\ \text { 10. } & \text { Jump from 3rd rank of } \mathbf{Z} \text { to } 1^{\text {st }} \text { rank of } & : & 1 \text { event } \\ \text { 11. } & \mathbf{Y} & : & 2 \text { events } \\ \text { 12. } & \text { Stay at 4th rank of } \mathbf{Y} & : & 3 \text { events } \\ \text { 13. } & \text { Stay at 3rd rank of } \mathbf{Y} & \text { Stay at 2nd rank of } \mathbf{Y} & \text { event } \\ \text { 14. } & \text { Stay at 1st rank of } \mathbf{Y} & : & 2 \text { events }\end{array}$

15.

Jump from 4th rank of $\mathbf{Z}$ to 3rd rank of

$$
\mathbf{X}
$$

Jump from 3rd rank of $\mathbf{Z}$ to 1 st rank of

$$
\mathbf{X}
$$

17.

18.

19.

20.

21.

22.

23.

24.

25.

26.

27.

28.

29.

Stay at 4th rank of $\mathbf{X}$

Stay at 3rd rank of $\mathbf{X}$

Stay at 2nd rank of $\mathbf{X}$

Stay at 1 st rank of $\mathbf{X}$

Jump from 4th rank of $\mathbf{Z}$ to 4 th rank of $\mathbf{Y}$

Jump from 4th rank of $\mathbf{Z}$ to 2nd rank of $\mathbf{X}$

Jump from 2nd rank of $\mathbf{Z}$ to 2nd rank of Y

Jump from 1st rank of $\mathbf{Z}$ to 1st rank of

$$
\mathbf{Y}
$$

Jump from 1st rank of $\mathbf{Z}$ to 1 st rank of$$
\mathbf{X}
$$

Jump from 3rd rank of $\mathbf{Z}$ to 3rd rank of Y

Jump from 3rd rank of $\mathbf{Z}$ to 3rd rank of $\mathbf{X}$

Jump from 2nd rank of $\mathbf{Z}$ to 2nd rank of $\mathbf{X}$

Jump from 2nd rank of $\mathbf{Z}$ to 1 st rank of $\mathbf{X}$

Then, the vector $\mathbf{W}, \mathbf{W}_{\mathbf{b}}$ for case 1-2, for example, are expressed as follows.

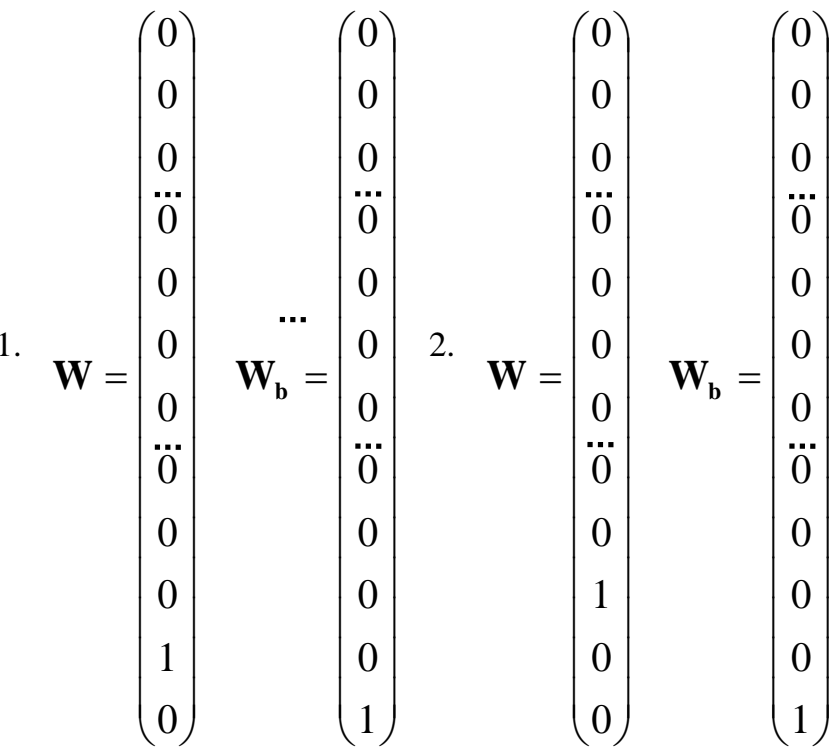

Substituting these to Eq.(18), we can obtain Eq.(26). 
$\mathbf{A}=\left(\begin{array}{cccc:cccc:cccc}1 & 0 & 0 & 0 & 0 & 0 & 0 & 0 & 1 & 1 & 1 & 0 \\ 0 & 3 & 0 & 0 & 0 & 0 & 0 & 0 & 0 & 2 & 0 & 0 \\ 0 & 0 & 2 & 0 & 0 & 0 & 0 & 0 & 0 & 0 & 1 & 2 \\ 0 & 0 & 0 & 3 & 0 & 0 & 0 & 0 & 0 & 0 & 0 & 2 \\ \hdashline 0 & 0 & 0 & 0 & 2 & -0 & 0 & 0 & 1 & 0 & -1 & -0 \\ 0 & 0 & 0 & 0 & 0 & 1 & 0 & 0 & 0 & 2 & 0 & 1 \\ 0 & 0 & 0 & 0 & 0 & 0 & 3 & 0 & 0 & 0 & 2 & 1 \\ 0 & 0 & 0 & 0 & 0 & 0 & 0 & 2 & 0 & 0 & 0 & 2 \\ \hdashline 0 & 0 & 0 & 0 & 0 & -0 & -0 & 0 & 2^{-} & 2^{-} & - & -0 \\ 0 & 0 & 0 & 0 & 0 & 0 & 0 & 0 & 0 & 4 & 0 & 1 \\ 0 & 0 & 0 & 0 & 0 & 0 & 0 & 0 & 0 & 0 & 4 & 2 \\ 0 & 0 & 0 & 0 & 0 & 0 & 0 & 0 & 0 & 0 & 0 & 3\end{array}\right) \times$

$\left(\begin{array}{cccc:cccc:cccc}1 & 0 & 0 & 0 & 0 & 0 & 0 & 0 & 0 & 0 & 0 & 0 \\ 0 & 3 & 0 & 0 & 0 & 0 & 0 & 0 & 0 & 0 & 0 & 0 \\ 0 & 0 & 2 & 0 & 0 & 0 & 0 & 0 & 0 & 0 & 0 & 0 \\ 0 & 0 & 0 & 3 & 0 & 0 & 0 & 0 & 0 & 0 & 0 & 0 \\ \hdashline 0 & 0 & 0 & 0 & 2 & 0 & 0 & 0 & 0 & 0 & 0 & 0 \\ 0 & 0 & 0 & 0 & 0 & 1 & 0 & 0 & 0 & 0 & 0 & 0 \\ 0 & 0 & 0 & 0 & 0 & 0 & 3 & 0 & 0 & 0 & 0 & 0 \\ 0 & 0 & 0 & 0 & 0 & 0 & 0 & 2 & 0 & 0 & 0 & 0 \\ \hdashline 0 & 0 & 0 & 0 & 0 & 0 & 0 & 0 & 4 & 0 & 0 & 0 \\ 0 & 0 & 0 & 0 & 0 & 0 & 0 & 0 & 0 & 11 & 0 & 0 \\ 0 & 0 & 0 & 0 & 0 & 0 & 0 & 0 & 0 & 0 & 9 & 0 \\ 0 & 0 & 0 & 0 & 0 & 0 & 0 & 0 & 0 & 0 & 0 & 14\end{array}\right)^{-1}$

(26)

$$
\left(\begin{array}{cccc:cccc:cccc}
1 & 0 & 0 & 0 & 0 & 0 & 0 & 0 & \frac{1}{4} & \frac{1}{11} & \frac{1}{9} & 0 \\
0 & 1 & 0 & 0 & 0 & 0 & 0 & 0 & 0 & \frac{2}{11} & 0 & 0 \\
0 & 0 & 1 & 0 & 0 & 0 & 0 & 0 & 0 & 0 & \frac{1}{9} & \frac{1}{7} \\
0 & 0 & 0 & 1 & 0 & 0 & 0 & 0 & 0 & 0 & 0 & \frac{1}{7} \\
\hdashline 0 & 0 & 0 & 0 & 1 & 0 & 0 & 0 & \frac{1}{4} & 0 & \frac{1}{9} & 0 \\
0 & 0 & 0 & 0 & 0 & 1 & 0 & 0 & 0 & \frac{2}{11} & 0 & \frac{1}{14} \\
0 & 0 & 0 & 0 & 0 & 0 & 1 & 0 & 0 & 0 & \frac{2}{9} & \frac{1}{14} \\
0 & 0 & 0 & 0 & 0 & 0 & 0 & 1 & 0 & 0 & 0 & \frac{1}{7} \\
\hdashline 0 & 0 & 0 & 0 & 0 & 0 & 0 & 0 & \frac{1}{2} & \frac{2}{11} & 0 & 0 \\
0 & 0 & 0 & 0 & 0 & 0 & 0 & 0 & 0 & \frac{4}{11} & 0 & \frac{1}{14} \\
0 & 0 & 0 & 0 & 0 & 0 & 0 & 0 & 0 & 0 & \frac{4}{9} & \frac{1}{7} \\
0 & 0 & 0 & 0 & 0 & 0 & 0 & 0 & 0 & 0 & 0 & \frac{3}{14}
\end{array}\right)
$$

Watching this, we can confirm following features as stated before. A of Eq.(21) became as follows.

$$
\mathbf{A}_{11}, \mathbf{A}_{22} \text { : Diagonal matrix }
$$

$$
\mathbf{A}_{12}, \mathbf{A}_{21}, \mathbf{A}_{31}, \mathbf{A}_{32}: \mathbf{0}
$$

$\mathbf{A}_{13}, \mathbf{A}_{23}, \mathbf{A}_{33}:$ Upper triangular matrix

Taking an automobile for example, customer brand selection from company A to B or company A to C can be made clear utilizing above stated method. We can confirm not only the preference shift among brands but also the preference shift among companies. This enables building marketing strategy for automobile company much easier.

\section{CONCLUSION}

Consumers often buy higher grade brand products as they are accustomed or bored to use current brand products they have.

In this paper, matrix structure was clarified when brand selection was executed toward higher grade brand. Expanding brand selection from single brand group to multiple genre brand group, we could make much more exquisite and multidimensional analysis. And formulation of extension to the brand groups was executed by using Block Matrix. $S$-step forecast model was also formulated. In numerical example, matrix structure's hypothesis was verified concerning brand selection among multiple brands. If we can see the trend of brand selection shift, we can foresee the brand selection shift towards another genre brand. Retailer can utilize the result of this to make effective marketing plan. Such research as 
questionnaire investigation of consumers' activity in automobile purchasing should be executed in the near future to verify obtained results.

\section{REFERENCES}

[1] Aker,D.A, Management Brands Equity, Simon \& Schuster, USA,1991.

[2] Katahira,H., Marketing Science (In Japanese), Tokyo University Press, 1987.

[3] Katahira,H.,Y,Sugita., "Current movement of Marketing Science" (In Japanese), Operations Research, 1994; 14: 178-188

[4] Takahashi,Y., T.Takahashi, "Building Brand Selection Model Considering Consumers Royalty to Brand"(In Japanese), Japan Industrial Management Association 2002; 53(5): 342-347

[5] Yamanaka,H., "Quantitative Research Concerning Advertising and Brand Shift" (In Japanese), Marketing Science, Chikra-Shobo Publishing, 1982.

[6] Takeyasu,K., Y.Higuchi, "Analysis of the Preference Shift of Customer Brand Selection among Multiple Genres of Automobile and Its Matrix Structure", Journal of Communication and Computer, 20012; 9(7): 744751

[7] Takeyasu,K., Y.Higuchi, “Analysis of the Preference Shift of Customer Brand Selection", International Journal of Computational Science, 2007; 1(4): 371-394 